\title{
Perceived impact and feasibility of strategies to improve access to healthy foods in Washington State, USA
}

\author{
Donna B Johnson ${ }^{1, *}$, Emilee L Quinn ${ }^{1}$, Mary Podrabsky' ${ }^{1}$, Nadia Beckwith-Stanley ${ }^{2}$, \\ Nadine Chan ${ }^{3}$, Amy Ellings ${ }^{4}$, Tricia Kovacs ${ }^{5}$ and Claire Lane ${ }^{6}$ \\ ${ }^{1}$ Center for Public Health Nutrition, University of Washington, Box 353410, Seattle, WA 98195 USA: \\ ${ }^{2}$ Children's Alliance, Seattle, WA, USA: ${ }^{3}$ Public Health Seattle and King County, Seattle, WA, USA: \\ ${ }^{4}$ Washington State Department of Health, Olympia, WA, USA: ${ }^{5}$ Washington State Department of Agriculture, \\ Olympia, WA, USA: ${ }^{6}$ WithinReach, Seattle, WA, USA
}

Submitted 15 November 2012: Final revision received 30 May 2013: Accepted 13 June 2013: First published online 7 August 2013

\begin{abstract}
Objective: The present study measured the perceived impact and political and implementation feasibility of state-level policy strategies related to increasing access to healthy foods and limiting unhealthy foods.

Design: Potential state-level policy strategies to improve access to healthy foods were identified through a review of evidence-based literature and policy recommendations. Respondents rated the perceived impact and political and implementation feasibility of each policy on a five-point scale using online surveys. Setting: Washington State policy process.

Subjects: Forty-nine content experts (national researchers and subject experts), forty policy experts (state elected officials or their staff, gubernatorial or legislative policy analysts) and forty-five other stakeholders (state-level advocates, programme administrators, food producers).

Results: In aggregate, respondents rated policy impact and implementation feasibility higher than political feasibility. Policy experts rated policy strategies as less politically feasible compared with content experts $(P<0 \cdot 02)$ or other stakeholders $(P<0 \cdot 001)$. Eight policy strategies were rated above the median for impact and political and implementation feasibility. These included policies related to nutrition standards in schools and child-care facilities, food distribution systems, urban planning projects, water availability, joint use agreements and breast-feeding supports.

Conclusions: Although they may be perceived as potentially impactful, some policies will be more difficult to enact than others. Information about the potential feasibility of policies to improve access to healthy foods can be used to focus limited policy process resources on strategies with the highest potential for enactment, implementation and impact.
\end{abstract}

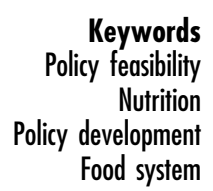

The public health goal to assure conditions in which people can be healthy ${ }^{(1)}$ requires that families and communities have sufficient access to healthy foods, defined in the USA as foods that can be used to meet the Dietary Guidelines for Americans ${ }^{(2)}$. Access to healthy foods has many dimensions including physical access (having food in geographic proximity given transportation options), economic access (having sufficient resources to pay for food), nutritional access (having access to food that is of good nutritional value) and cultural access (having food that is culturally appropriate $)^{(3-5)}$.

In their efforts to assure access to healthy foods, public health nutritionists join a broad group of stakeholders who work to improve the capacity of food systems to deliver healthy foods to the population ${ }^{(6,7)}$. Nutritional status and access to healthy foods are impacted by many factors that interact in complex systems; sustainable initiatives to assure such access will require multidimensional changes across a broad set of food access determinants ${ }^{(7,8)}$. These changes can be supported by a 'health in all ${ }^{(9)}$ approach to developing, enacting and implementing effective policies to support actions at each point in the food system from production, transformation, distribution, retail and consumption through to waste management ${ }^{(7)}$. Such policies make it easier to make healthy lifestyle choices ${ }^{(10)}$, but they require strong leadership and substantial public policy change ${ }^{(11)}$.

An impressive list of policy strategies to improve food systems and increase access to healthy foods has been generated by researchers, practitioners, advocates and 
other policy actors. These include a wide range of legislative, regulatory and procedural approaches such as making food systems more health promoting overall ${ }^{(12)}$, planning communities to assure food access for residents ${ }^{(13)}$, promoting healthy environments in schools ${ }^{(14)}$, promoting breast-feeding in hospitals and communities ${ }^{(15)}$, establishing tax policy incentives and disincentives ${ }^{(16)}$ and addressing family food security ${ }^{(17)}$. Because this array of policies can be overwhelming to policy makers, there is a need to provide policy makers with concise information about opportunities for positive change ${ }^{(6)}$.

Public health practitioners are accustomed to seeking out evidence about what works to improve health, but the health research literature offers little evidence about the process of developing and implementing policy in a state or local context ${ }^{(17)}$. Most policies put forth by wellmeaning advocates are not enacted ${ }^{(14)}$. Policy development is subject to political and social influences, and policy makers are subject to many competing demands ${ }^{(18)}$. Little is known about stakeholder perceptions of policy impact, the feasibility of policy adoption or implementation, or how these perceptions are formed. For example, a diverse set of policy actors (advocates, academics, elected officials and agency staff) often come together around a given policy, so it is important to understand the beliefs of each of these actors about the dimension of policy that are most important to them ${ }^{(18,19)}$. Policy process research and a better understanding of the policy landscape can inform the steps of policy formulation, enactment and implementation ${ }^{(20)}$. Most major policy shifts require several years of preparatory action ${ }^{(19)}$; identifying early 'easy wins' can support comprehensive long-term efforts to assure access to healthy foods ${ }^{(21)}$.

In 2010, the Washington State Nutrition and Obesity Policy Research and Evaluation Network (WA NOPREN), funded by the Centers for Disease Control and Prevention (CDC) as part of a national NOPREN to conduct 'transdisciplinary nutrition policy research', served as an advisory group for the development of a report on Opportunities for Increasing Access to Healthy Foods In Washington $^{(22)}$ (referred to hereafter as the 'Opportunities report'). WA NOPREN members have expertise in nutrition, agriculture, public health, food systems, law, economics, public policy, epidemiology, evaluation and urban design. The group includes representatives from state and local health departments, other state agencies, policy and advocacy organizations, and the state's universities. The Opportunities report was based on a summary of the status of the current food system in the state, interviews with sixty-five state leaders and experts in food and economic systems, and a review of over 100 policy recommendation and guidance documents. While policy strategies are recommended at international, national and local levels, this report focused only on state actions. Results of the needs assessment, interviews and document review were compiled to develop a list of the highest-priority strategies for improving healthy food access in families, communities, institutions and regional food systems.

The purpose of the present study is to build on the Opportunities report's findings to further inform policy development initiatives to increase access to healthy foods. The study addresses three research questions:

1. To what extent are specific state-level policy strategies to improve access to healthy foods perceived as impactful, politically feasible and implementable?

2. Do content experts, policy experts and other stakeholders differ in their perceptions of the impact and feasibility of policy strategies to improve access to healthy foods?

3. Which food policy strategies are most promising in terms of their impact and feasibility?

The study can impact public health interventions by identifying the most promising strategies for public health practitioners to pursue. Practitioners can apply the methodology described here in other settings.

\section{Methods}

Data were collected through a sequential series of anonymous online surveys completed by national and state nutrition and food system content experts, Washington State researchers and food system stakeholders, and Washington State policy experts. Table 1 provides a summary of recruitment procedures and sample size for each of the respondent groups. The content expert and other stakeholder samples were developed using an initial roster of members of the national NOPREN network of universitybased researchers and the WA NOPREN. These individuals, in turn, nominated content experts and other stakeholders.

\section{Policy strategy selection}

The list of policies included in the survey was based on a framework from the Opportunities report ${ }^{(22)}$. While there is overlap in policies designed to address food access and obesity prevention, the primary focus of the report is healthy food access. These categories included: Food Marketing; Price Incentives; Access to Food Retailers; Community Planning and Land Use; Nutrition in ChildCare Facilities; Nutrition in Schools; State Agency Nutrition Standards; Local Food Procurement; Farmer Support and Agricultural Preservation; Breast-feeding; and Other (i.e. water access and joint use agreements). The team then further reviewed extant literature, primarily in the form of practice and policy guides, to identify specific state-level policy strategies that are recommended for each category ${ }^{(23-26)}$. The WA NOPREN advisory group provided additional ideas for policy solutions and helped to prioritize and edit the list based on its knowledge of Washington's unique context. For example, some of the 
policy statements related to nutritional standards of foods served in government facilities came from a current draft of a proposed state food procurement bill. Policy strategies currently in place in Washington in some form were either not included or were revised to describe a stronger policy approach. Policies related to Farm-toSchool programming were not included because they were already part of the state's Local Farms Healthy Kids Act passed two years prior. In another case, a policy statement related specifically to the funding of a state breast-feeding coordinator, since the position is mentioned, but not funded in existing state policy.

\section{Online surveys}

Surveys began with a statement that described 'healthy food access' broadly, inclusive of physical, financial, nutritional and cultural dimensions. The online surveys included questions and associated Likert-scale response options for the perceived impact, political feasibility and implementation feasibility (referred to hereafter as 'dimensions') of each policy statement. Survey questions were:

1. 'To what extent would this policy positively impact the population's access to healthy foods?'

2. 'To what extent could this policy be adopted or enacted within the next 5 years?'

3. 'To what extent could this policy be implemented as described if adopted or enacted?'

Responses for each of the three questions included a 5-point scale of 'no impact or feasibility' (score $=0$ ), 'minimal' (score $=1)$, 'medium' (score $=2)$, 'considerable' (score $=3$ ) and 'maximum impact or feasibility' (score $=4$ ). Respondents also had the option to select 'I don't know'. Respondents identified their primary role with regard to the food system. The research team used these responses to categorize respondents into three groups for analysis: (i) content experts (including both national and Washington State nutrition and food system subject experts, predominantly researchers); (ii) policy experts (Washington State elected officials or their staff, gubernatorial or legislative policy analysts); and (iii) other Washington food system stakeholders (advocates, programme administrators, food producers).

Data collection took place between May and September 2011. Since the overall study design called for data on three dimensions for each of fifty policies, the research team designed a pragmatic data collection process to minimize the burden on individual respondents while still providing data useful to state planners. First, some respondents were asked to rate only the dimension(s) most pertinent to their perspective. Because they possessed less familiarity with Washington's political and implementation landscape, national content experts were only asked to rate the policies' potential impact. Washington State policy experts were only asked to rate policies' political feasibility, acknowledging that brevity was critical to ensure responses from busy policy makers - and that policy experts possess unique authority to assess this dimension. All other respondents (Washington State content experts and 'other stakeholders') were asked to rate policy strategies across all three dimensions. In addition, the research team used results from successive data collection rounds to eliminate low rated policies early on and keep the surveys as short as possible for subsequent rounds of data collection. Content experts from outside the state, those with the least stake in a process conducted for Washington State, received the survey first and the research team removed the ten policies that this group rated lowest impact before opening the survey to other respondent groups. Removed policies included, for example, developing and mandating a standards-based nutrition programme in schools, mandating menu labelling at chain restaurants with between ten and twenty locations, and providing a transportation subsidy to Supplemental Nutrition Assistance Program recipients. Washington State content experts and other stakeholders responded next, after which the research team removed an additional three policy strategies that scored low for all dimensions before opening the survey to Washington policy experts.

Survey invitation and reminder protocols were based on Dillman's recommendations ${ }^{(27)}$. An initial packet of materials that included an introduction to the project and its sponsors and the executive summary of the Opportunities report was mailed to policy experts. This was followed by an emailed invitation to participate in the survey. Postcard reminders were sent to policy experts who did not respond in the first week, and an attempt was made to contact each non-responsive policy expert by telephone. Content experts and other stakeholders were invited to participate through emailed invitations. Reminders were emailed to those who did not respond to the invitations about weekly for up to five weeks after the initial invitation.

For policy experts, the research team asked that either a legislator or his or her staff complete the survey. In instances when both completed the survey, only the legislator's response was included in analysis. As an incentive to participate, all content experts and other stakeholder survey participants were entered into a prize draw. Policy experts did not receive any incentives. The surveys took an estimated 15-45 min to complete. The study was conducted according to the guidelines laid down in the Declaration of Helsinki, and all procedures involving human subjects/patients were reviewed by the University of Washington Human Subjects Division which determined that the project did not require full review by the institutional review board because it qualified for exempt status.

\section{Data analysis}

Statistical analyses were completed with the statistical software package IBM SPSS Statistics 19. Descriptive statistics 
were used to calculate frequencies for demographic data and mean ratings for each feasibility dimension and policy statement. Scatter plots and Pearson's correlation coefficients were generated to examine relationships between mean policy ratings of each respondent group pairing (e.g. content experts and policy experts) and dimension pairing (e.g. impact and political feasibility), and ANOVA with Tukey corrections for multiple comparisons was used to assess differences between responses by respondent groups. The research team used the median policy rating for each dimension to classify mean ratings above the median as 'higher' impact or feasibility and those below the median as 'lower' impact or feasibility.

\section{Results}

\section{Sample}

Of 386 individuals or offices invited to participate, 134 responded to the online survey. The sample is described in Table 1 . Of the forty policy experts, eleven were state legislators, twenty-four were legislative staff and five were legislative policy analysts. Most of the policy experts who participated were from the highly populated urban parts of the state.

\section{Policy ratings}

As indicated by the data in Table 2, on a scale from 0 to 4 , respondents rated the policies in aggregate as slightly higher than 'medium' for impact and implementation feasibility, and slightly lower than 'medium' for political feasibility. For individual policy statements, mean ratings ranged between 2.04 and 2.85 for impact, between 1.32 and 2.71 for political feasibility, and between 1.89 and 3.00 for implementation feasibility.

Figure 1 illustrates that the overall trend for individual policy options was to have lower ratings for political feasibility than impact and implementation feasibility. The exceptions to this pattern were policy strategies to ban advertising on school buses and school grounds. Scatter plots in Fig. 2 show relationships between the three dimensions for each of the policy strategies. Political and implementation feasibility were significantly associated $(P<0 \cdot 01)$; neither political feasibility and impact nor impact and implementation feasibility were associated.

Overall, about $10 \%$ of respondents selected 'I don't know' for a given policy statement instead of rating its impact and/or feasibility. The fewest 'I don't know' responses were for policies related to food marketing, price incentives, food retail, school nutrition and state agency standards. The most 'I don't know' responses were for policies related to child-care standards, farmer support and agriculture, and breast-feeding. More than $20 \%$ of respondents selected 'I don't know' for these three policies:

1. Issue additional state-determined nutrition standards for participation in the US Department of Agriculture's

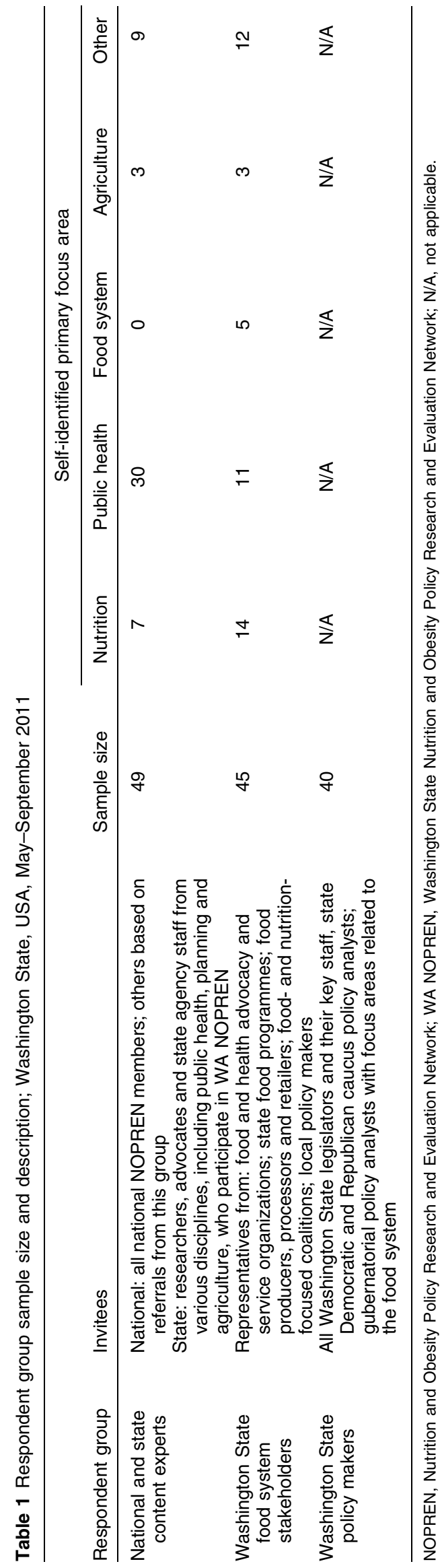


Table 2 Mean rating of thirty-seven policies' perceived impact and feasibility by respondent group; respondents to an online survey on policy strategies to improve access to healthy foods, Washington State, USA, May-September 2011

\begin{tabular}{|c|c|c|c|c|c|c|c|c|}
\hline & \multicolumn{2}{|c|}{ National and state content experts } & \multicolumn{2}{|c|}{ Other stakeholders } & \multicolumn{2}{|c|}{ Policy experts } & \multicolumn{2}{|c|}{ All respondents } \\
\hline & $n$ & Mean & $n$ & Mean & $n$ & Mean & $n$ & Mean \\
\hline Impact & 49 & $2 \cdot 37$ & 45 & $2 \cdot 53$ & - & - & 94 & $2 \cdot 45$ \\
\hline Political feasibility & 16 & $2 \cdot 15$ & 44 & $2 \cdot 15$ & 40 & 1.57 & 100 & $1 \cdot 91$ \\
\hline Implementation feasibility & 16 & $2 \cdot 32$ & 44 & $2 \cdot 36$ & - & - & 60 & $2 \cdot 34$ \\
\hline
\end{tabular}

Scale: $0-4 ; 0=$ none, $1=$ minimal, $2=$ medium, $3=$ considerable, $4=$ maximum.

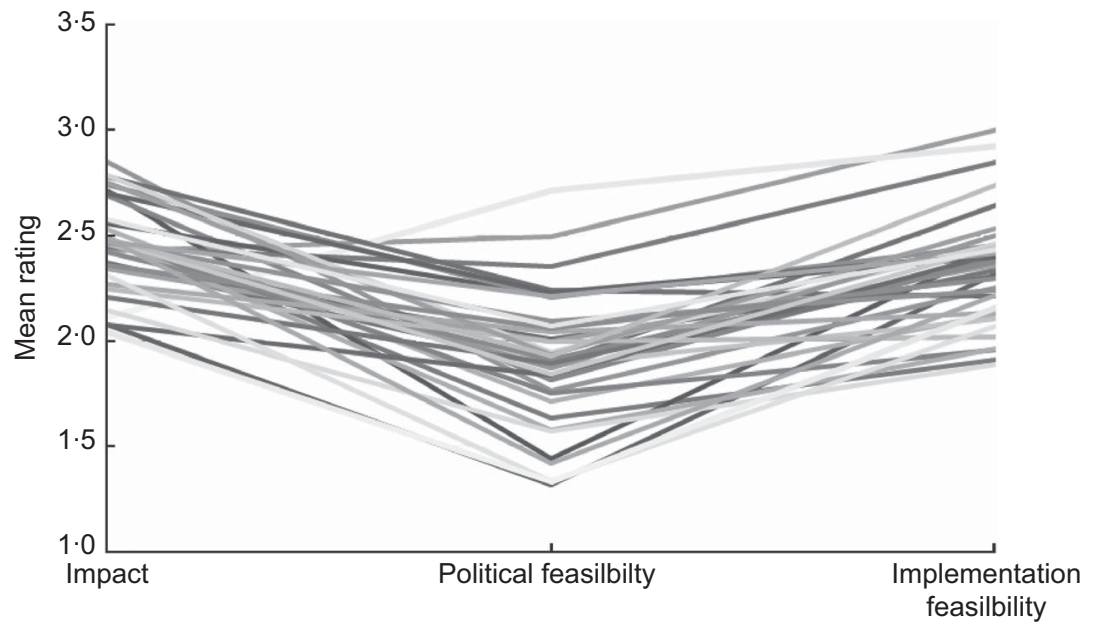

Fig. 1 Mean impact, political feasibility and implementation feasibility ratings (on a scale from 0 to 4 ) among respondents to an online survey on policy strategies to improve access to healthy foods, Washington State, USA, May-September 2011

Child and Adult Care Food Program (CACFP) above and beyond the federal minimum standards; twenty-nine 'don't knows'.

2. Ban restrictive land covenants that do not allow new grocery chains to move into locations previously held by another chain; thirty-eight 'don't knows'.

3. Levy taxes on the conversion to non-farm uses of land designated for agricultural land preservation; thirtyeight 'don't knows'.

\section{Policy ratings - differences by respondent group}

Table 2 presents the ratings of each dimension overall and by stakeholder group. Policy experts rated the policies as less politically feasible than content experts $(P<0 \cdot 018)$ or other stakeholders $(P<0 \cdot 001)$. The biggest differences in political feasibility ratings were for strategies that addressed funding and support for healthy eating media campaigns, encouraging local communities to address healthy food access in transportation and land-use planning, applying standards for trans fat in food at government facilities, funding farmer business training and funding a breast-feeding coordinator. There were no statistically significant differences in impact or implementation ratings between respondent groups.

\section{Highest ranked strategies across the dimensions}

Using the median of each dimension as the cut-off ( median $=2.47$ for impact, median $=1.91$ for political feasibility and median $=2 \cdot 36$ for implementation feasibility), Table 3 presents policy ratings for each dimension, categorized as rated relatively 'high' or 'low' in like combination groupings. Eight policies achieved ratings above the median for all three of the dimensions. The top three rated policies for perceived impact were provide financial and technical support to Washington public schools in adhering to the most recent nutrition standards released by the US Department of Agriculture (2.782), followed by fund pilot projects to build regional and local food distribution systems $(2 \cdot 750)$ and make participation in the federal CACFP a standard of quality for child-care, early learning and after-school programmes $(2 \cdot 738)$. The top three rated policies for political feasibility were create consistent nutritional standards across various forms of licensed child care $(2 \cdot 227)$, followed by encourage cities and counties to address access to farmers' markets, community-supported agriculture and farmable land in state-administered urban planning grants $(2 \cdot 220)$ and broaden state regulations to expressly permit and address liability issues of joint use agreements to be used for community purposes, such as community kitchens $(2 \cdot 212)$. Finally, the top three rated policies for implementation feasibility were cover breast pumps rentals or purchases for working mothers through state-funded health insurance $(2 \cdot 740)$, followed by fund pilot projects to build regional and local food distribution systems (2.537) 

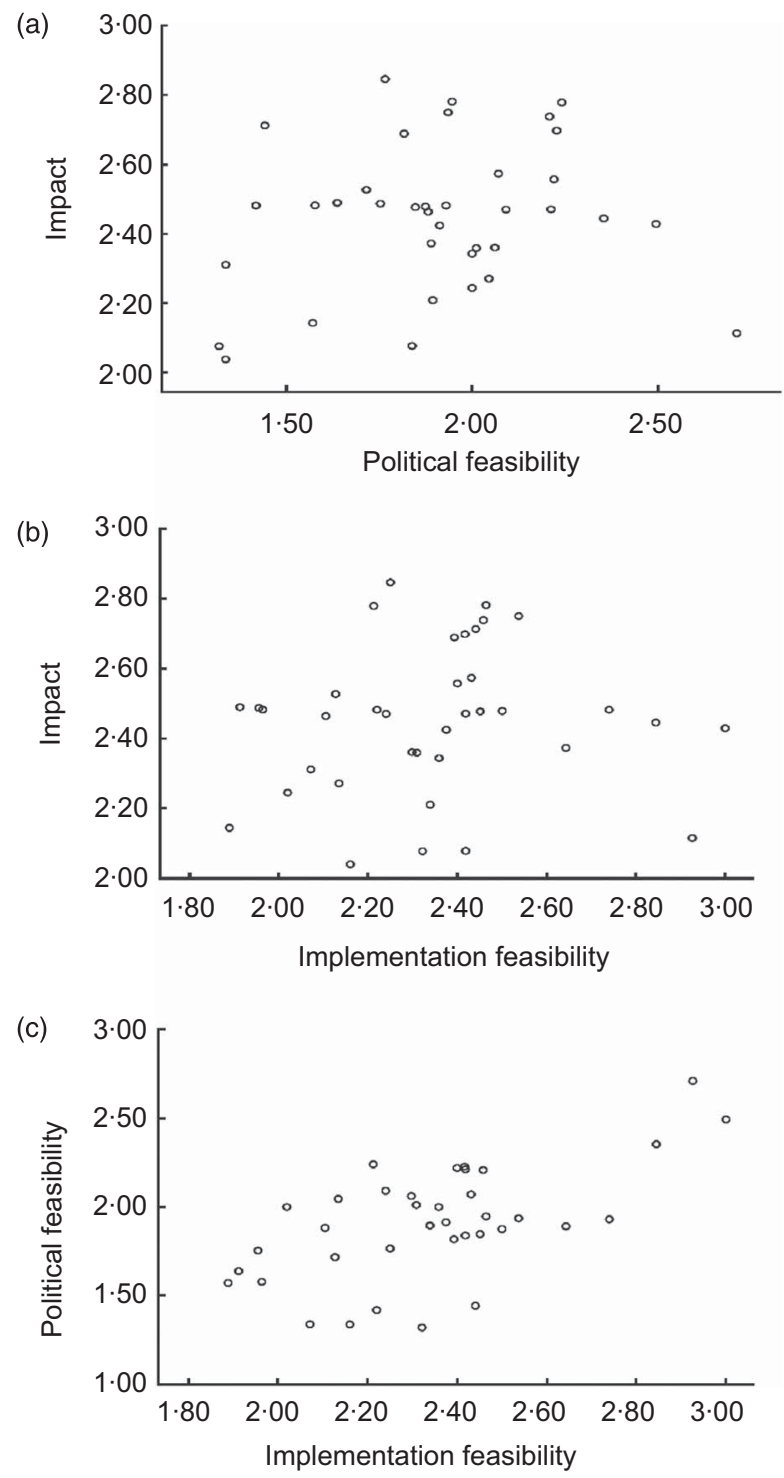

Fig. 2 Scatter plots of mean policy ratings (on a scale from 0 to 4), comparing dimension pairings of (a) impact and political feasibility, (b) impact and implementation feasibility and (c) political feasibility and implementation feasibility, among respondents to an online survey on policy strategies to improve access to healthy foods, Washington State, USA, MaySeptember 2011

and make participation in the federal CACFP a standard of quality for child-care, early learning and after-school programmes $(2 \cdot 458)$.

\section{Discussion}

Information about the potential feasibility and effectiveness of nutrition- and food-related policy change can be used to focus limited policy process resources on those policy strategies with the best chances of successful enactment, implementation and impact. While the present study focused on state-level strategies, similar studies have been conducted in local communities ${ }^{(28,29)}$ and internationally with stakeholders from nine European Union countries ${ }^{(30)}$.

In the current study, most policy strategies were seen as more impactful and feasible for implementation than politically feasible. The eight diverse policies that scored higher than the median on all three dimensions related to school and child-care standards, local food systems and community planning, water access, joint use, and breastfeeding. A number of the policies rated above the median were framed as approaches to encourage and support rather than mandate changes. Specific regulation changes that are within accepted parameters for governmental actions, such as insurance coverage for breast pumps and explicit options for joint use of publicly owned properties like school gymnasiums, may be perceived as relatively non-controversial and non-costly, technical changes to make in state policy.

Other studies have reported similar results. In a study of childhood obesity policy strategies, Brescoll and colleagues $^{(31)}$ also found that content experts ranked policies higher for potential impact $(4 \cdot 27)$ than policy makers did for political feasibility (3.48). Childhood obesity policies that were viewed as potentially politically feasible but not very impactful included informational approaches such as providing parents and children with nutrition information about school lunches and increasing federal funding for nutrition education. A report on interviews with forty-five senior governmental and non-governmental authorities in Australia ${ }^{(32)}$ found that the highest level of support was expressed for policies that addressed children or fell within traditional governmental boundaries such as school nutrition and cooking classes and nutrition standards for foods in government institutions including schools, prisons and government offices. For childhood obesity in the USA, strategies that involve outright bans and prohibitions were rated as impactful, but not politically feasible $^{(31)}$, and in Australia policy strategies that were outside educational or conventional regulatory arenas, such as those that addressed food production, distribution and retail, received the least support ${ }^{(32)}$.

Perceptions about political feasibility may be negatively affected by the perceived influence of those who may oppose policy change. An analysis of national nutrition policies in New Zealand found that current policies are more aligned with industry interests than with public health ${ }^{(33)}$. In the current study, a tax on sugarsweetened beverages (SSB) was categorized as more impactful by content experts but as less feasible by the policy experts and other stakeholders. This perception about a lack of political feasibility for SSB taxes is supported by findings that only $36 \%$ of US adults support a tax on $\mathrm{SSB}^{(34)}$. In Washington State, the perception of low political feasibility was probably enhanced by the recent passage of a voter initiative that repealed a very modest tax on nutrient-poor/energy-dense foods and beverages. 
Table 3 Mean policy ratings for impact, political feasibility and implementation feasibility categorized by higher (above median) and lower (below median)* among respondents to an online survey on policy strategies to improve access to healthy foods, Washington State, USA, May-September 2011

Policy
High impact/high political feasibility/high implementation feasibility
Provide financial and technical support to Washington public schools in adhering to the most recent nutrition standards
released by USDA
Fund pilot projects to build regional and local food distribution systems between Washington farmers, institutions and
food retailers
Make participation in the federal CACFP a standard of quality for child-care, early learning and after-school programmes
Create consistent nutritional standards across various forms of licensed child care
Require that plain water be available in government-operated and -administered outdoor areas and other public places and facilities
Encourage cities and counties to address access to farmers' markets, CSA and farmable land in state-administered urban
planning grants
Cover breast pumps rentals or purchases for working mothers through state-funded health insurance
Broaden state regulations to expressly permit and address liability issues of joint use agreements for schools and other
government facilities to be used for community purposes, such as community kitchens
High impact/low political feasibility/high implementation feasibility

Develop a state-wide public-private partnership to finance the development of supermarkets and fresh food retailers in low-income communities

Provide tax incentives for grocery stores that locate in low-income communities

Establish small business assistance programmes to support small corner convenience stores in selling affordable healthy food (e.g. training and equipment to sell perishable items)

Issue additional state-determined nutrition standards for participation in the USDA CACFP above and beyond the federal minimum standards

Establish nutritional standards for the sale of foods and beverages in settings frequented by children (e.g. zoos, children's museums and recreation venues)

Mandate that all Washington public schools participate in the HealthierUS Schools Challenge

Fund Breastfeeding Friendly Worksite Program (currently in state law but with no funding)

High impact/high political feasibility/low implementation feasibility

Revise current child-care licensing regulations for nutrition to align with evidence-based standards and national guidelines

High impact/low political feasibility/low implementation feasibility

Institute a tax of 2 cents/ounce on SSB and designate the revenue for obesity prevention among children and adults

Fund Farm-to-School projects to fund to increase the use of local foods in school meals

Fund business training for farmers to respond to the emerging market for local food/farm products

Low impact/high political feasibility/high implementation feasibility

Fund additional technical support for farmers' markets in acquiring and using EBT terminals to accept SNAP and WIC benefits Prohibit the advertising of foods or beverages in school buildings or on school grounds except for those meeting

nutritional standards

Mandate that lunches served by state agencies include at least two fruits and vegetables

Ban restrictive land covenants that do not allow new grocery chains to move into locations previously held by another chain Prohibit food and beverage advertising on school buses

Low impact/high political feasibility/low implementation feasibility

Establish purchasing incentives for state agency procurement through local farmer cooperatives

Provide technical assistance for the formation of agricultural cooperatives

Phase in requirements that all farmers markets accept EBT cards

Require that state agencies give preference to a provider of locally grown food when the cost of such food is not more than $10 \%$ higher than the lowest bid for a procurement contract

\begin{tabular}{|c|c|c|c|c|c|}
\hline \multicolumn{2}{|c|}{ Impact } & \multicolumn{2}{|c|}{$\begin{array}{l}\text { Political } \\
\text { feasibility }\end{array}$} & \multicolumn{2}{|c|}{$\begin{array}{l}\text { Implementation } \\
\text { feasibility }\end{array}$} \\
\hline$n$ & Mean & $n$ & Mean & $n$ & Mean \\
\hline
\end{tabular}

列 to be grown, packaged or processed in Washington

\begin{tabular}{|c|c|c|c|c|c|}
\hline 87 & $2 \cdot 78$ & 93 & 1.95 & 56 & $2 \cdot 46$ \\
\hline 84 & $2 \cdot 75$ & 93 & 1.94 & 54 & 2.54 \\
\hline 84 & $2 \cdot 74$ & 77 & $2 \cdot 21$ & 48 & $2 \cdot 46$ \\
\hline 86 & $2 \cdot 70$ & 88 & $2 \cdot 23$ & 48 & $2 \cdot 42$ \\
\hline 89 & $2 \cdot 57$ & 85 & $2 \cdot 07$ & 51 & $2 \cdot 43$ \\
\hline 88 & $2 \cdot 56$ & 91 & $2 \cdot 22$ & 55 & $2 \cdot 40$ \\
\hline 81 & $2 \cdot 48$ & 86 & 1.93 & 50 & $2 \cdot 74$ \\
\hline 85 & $2 \cdot 47$ & 85 & $2 \cdot 21$ & 55 & $2 \cdot 42$ \\
\hline 91 & $2 \cdot 85$ & 94 & $1 \cdot 77$ & 52 & $2 \cdot 25$ \\
\hline 90 & $2 \cdot 69$ & 93 & $1 \cdot 82$ & 56 & $2 \cdot 39$ \\
\hline 93 & 2.53 & 95 & $1 \cdot 72$ & 55 & $2 \cdot 13$ \\
\hline 76 & $2 \cdot 49$ & 73 & $1 \cdot 75$ & 45 & 1.96 \\
\hline 94 & $2 \cdot 49$ & 91 & $1 \cdot 64$ & 57 & 1.91 \\
\hline 83 & $2 \cdot 48$ & 90 & 1.58 & (55) & 1.96 \\
\hline 81 & $2 \cdot 48$ & 86 & 1.42 & 50 & $2 \cdot 22$ \\
\hline 86 & $2 \cdot 78$ & 83 & $2 \cdot 24$ & 47 & $2 \cdot 21$ \\
\hline 94 & $2 \cdot 71$ & 95 & $1 \cdot 44$ & 59 & $2 \cdot 44$ \\
\hline 92 & $2 \cdot 48$ & 96 & $1 \cdot 88$ & 58 & $2 \cdot 50$ \\
\hline 86 & $2 \cdot 48$ & 85 & 1.85 & 51 & $2 \cdot 45$ \\
\hline 90 & $2 \cdot 44$ & 96 & $2 \cdot 35$ & 58 & $2 \cdot 84$ \\
\hline 91 & $2 \cdot 43$ & 89 & $2 \cdot 49$ & 55 & 3.00 \\
\hline 92 & $2 \cdot 42$ & 92 & 1.91 & 56 & $2 \cdot 38$ \\
\hline 70 & $2 \cdot 34$ & 71 & $2 \cdot 00$ & 39 & $2 \cdot 36$ \\
\hline 88 & $2 \cdot 11$ & 90 & $2 \cdot 71$ & 54 & 2.93 \\
\hline 83 & $2 \cdot 47$ & 88 & 2.09 & 50 & $2 \cdot 24$ \\
\hline 75 & $2 \cdot 36$ & 82 & $2 \cdot 06$ & 47 & $2 \cdot 30$ \\
\hline 92 & $2 \cdot 36$ & 90 & $2 \cdot 01$ & 55 & $2 \cdot 31$ \\
\hline 85 & $2 \cdot 27$ & 89 & $2 \cdot 04$ & 52 & $2 \cdot 13$ \\
\hline 78 & $2 \cdot 24$ & 88 & $2 \cdot 00$ & 51 & $2 \cdot 02$ \\
\hline
\end{tabular}


The beverage industry funded the voter initiative heavily and it is likely that the perceived influence of industry opposition to an SSB tax is behind the low political feasibility of this strategy, despite its perceived impact.

In contrast to the perceived relative lack of political support for strategies to reduce access to unhealthy foods such as taxing SSB, there does seem to be support for strategies to increase access to fruits and vegetables. While it was not seen as especially impactful, support for building the infrastructure to allow use of electronic benefit technology for food assistance programme beneficiaries at farmers' markets was seen as politically feasible. Foltz et $a l .{ }^{(35)}$ recently reported that $62 \%$ of the general public supports changes to state or local policies to improve fruit and vegetable access through farmers' markets.

The complexity of food and nutrition systems strategies may be a barrier to policy development and implementation. Many strategies in the current study had fairly high numbers of 'I don't know' responses. Allender and co-workers ${ }^{(36)}$ reported substantially more support for policy changes to support physical activity than healthy eating in Australia, and hypothesized that policy makers could easily understand the need for physical activity facilities but had difficulty understanding the more complex systems changes needed to address the food supply and food marketing. Food procurement policies may also be hindered by their perceived complexity. Gase et al. ${ }^{(37)}$ found that lack of knowledge and experience was a barrier to implementing sodium procurement standards.

While the present study provides insight into the perceptions of policy actors for access to healthy foods, it has limitations. The policy strategies were selected based on their relevance to Washington State's context; perceptions may differ in other states. Respondents who chose to participate may have had characteristics distinct from those who did not; those who participated may be more interested in or generally supportive of policy approaches to address the issue of healthy food access than those who chose not to complete the survey. Few representatives of food industry, in particular, participated in the study and the policy expert response rate was somewhat lower than previously reported average response rates for legislator surveys of comparable length ${ }^{(38)}$. The diverse sample included individuals from various sectors of the food system, so they may not have been knowledgeable about all of the policy approaches. Based on use of the 'I don't know' response patterns, respondents felt more capable of rating some policies (e.g. food marketing) than others (e.g. child-care standards). In an intentional effort to make the data as useful as possible for Washington State policy efforts to increase access to healthy foods, the research team focused on the goals of creating a list of priority policy strategies and reducing respondent burden. Because policy experts were not asked to rate all policies on each dimension, it was not possible to assess policy experts' perceptions of policy impact or implementation, or compare 
these opinions with those of other stakeholders. While all respondents were not asked all questions, there was an attempt to capture the most important perceptions according to the actors' roles in the policy system ${ }^{(18)}$. Only sixteen of the content expert respondents were from Washington, so this group was somewhat small in comparison with the number of stakeholders ( $n$ 44) and policy makers ( $n$ 40) asked to rate the policies' political feasibility in the state. Some policies were removed from the survey early in data collection. As a result, the findings reflect the extent to which policies were seen as highly impactful or feasible, but not necessarily which policies are seen as the least impactful or feasible. The policy matrix divides the policies by ratings relative to one another to aid in policy prioritization. The actual range of mean ratings was small and few policies emerged as rated considerably higher than others. In fact, all thirty-seven policies achieved a mean rating of at least 'medium' impact and most policies did so related to implementation feasibility. The calculation of mean rating scores in effect masks heterogeneity of opinions across the sample. Asking respondents to identify preferred policies or rank the options may be a better approach when a shorter list of policies is being considered.

It is a strength that the study's participatory design and attention to state context have resulted in useful and practical information. Although the policy list was not intended to be comprehensive of all possible state-level strategies to increase access to healthy foods, the list was compiled based on prior state reports and input from state stakeholders. A wide variety of state stakeholders was invited to participate in the study. Perhaps the most notable observation to be made is that no one policy category emerged as the clear favourite; respondents feel that a mix of policies pertaining to sectors across the food system is needed and feasible.

There are several opportunities to further this line of study, including conducting studies in states with different demographic and political profiles. In addition, it would be helpful to gather qualitative information about the factors that respondents consider when they are ranking the policies. What is the role of evidence in decision making? What priorities drive interest in access to healthy foods? What roles do community and leadership support play in policy feasibility perceptions? For example, in Washington State, the fact that an SSB tax with proceeds dedicated to obesity prevention efforts was rated as more impactful than a tax without this specification indicates that respondents may have been interested in policy influence on unhealthy as well as healthy foods. Finally, it would be useful to crosswalk the findings presented here with evidence of effectiveness and actual policy adoption and implementation rates.

The present study provides useful insight into the perceived impact and feasibility of a wide array of statelevel strategies to increase access to healthy foods. Since the study was conducted in Washington State, findings were shared with decision makers involved in a statelevel food system planning process and significant progress has been made in three of the top eight rated policies. These methods can be used in other settings to provide a short list of policies considered most impactful and feasible, relative to others, that policy makers and planners can consider for early action. Furthermore, aggregate scores based on different weights of the three dimensions could be constructed to inform decisions based on stakeholders' relative interest in the degree to which policies are impactful or feasible. A process such as this one could also serve as an early-stage prioritization exercise to take into account other critical considerations, such as impact on equity. Public health practitioners can also increase likelihood of policy success by thinking about each of the three dimensions measured here and fully considering policy makers' scepticism about political feasibility.

\section{Acknowledgements}

Sources of funding: This paper was supported through NOPREN by Cooperative Agreement Number 5U48DP001911 from the CDC. The findings and conclusions in this paper are those of the authors and do not necessarily represent the official position of the CDC. CDC had no role in the design, analysis or writing of this article. Conflicts of interest: No conflicts of interest exist. Authors' contributions: All of the authors contributed to the design of this study. D.B.J. was the principal investigator of this project, and E.L.Q. and M.P. coordinated data collection activities. D.B.J. and E.L.Q. wrote paper drafts, and all authors contributed to review and paper finalization. Acknowledgements: The authors would also like to acknowledge members of the WA NOPREN, particularly Erin MacDougall, Victor Colman, Marilyn Sitaker, Laurie Anderson, Deborah Allen, Linda Stone and Patricia Lichiello, as well as the national NOPREN members, for their contributions.

\section{References}

1. Institute of Medicine, Committee on the Future of Public Health (1988) The Future of Public Health. Washington, DC: National Academy Press.

2. US Department of Health and Human Services \& US Department of Agriculture (2010) Dietary Guidelines for Americans, 2010, 7th ed. Washington, DC: Government Printing Office.

3. Rose D (2010) Access to healthy food: a key focus for research on domestic food insecurity. J Nutr 140, 1167-1169.

4. Renzaho AM \& Mellor D (2010) Food security measurement in cultural pluralism: missing the point or conceptual misunderstanding? Nutrition 26, 1-9.

5. Gittelsohn J \& Sharma S (2009) Physical, consumer, and social aspects of measuring the food environment among 
diverse low-income populations. Am J Prev Med 36, 4 Suppl., S161-S165.

6. Story M, Hamm MW \& Wallinga D (2009) Research and action priorities for linking public health, food systems, and sustainable agriculture: recommendations from the Airlie Conference. J Hunger Environ Nutr 4, 477-485.

7. Muller M, Tagtow A, Roberts SL et al. (2009) Aligning food systems policies to advance public health. J Hunger Environ Nutr 4, 225-240.

8. Sacks G, Swinburn B \& Lawrence M (2009) Obesity policy action framework and analysis grids for a comprehensive policy approach to reducing obesity. Obes Rev 10, 76-86.

9. Mayes R \& Oliver TR (2012) Chronic disease and the shifting focus of public health: is prevention still a political lightweight? J Health Polit Policy Law 37, 181-200.

10. Swinburn BA, Sacks G, Hall KD et al. (2011) The global obesity pandemic: shaped by global drivers and local environments. Lancet 378, 804-814.

11. Gortmaker SL, Swinburn BA, Levy D et al. (2011) Changing the future of obesity: science, policy, and action. Lancet 378, 838-847.

12. American Public Health Association (2007) Toward a Healthy Sustainable Food System. http://www.apha.org/advocacy/ policy/policysearch/default.htm?id=1361 (accessed January 2011).

13. American Planning Association (2007) Policy Guide on Community and Regional Food Planning. http://www. planning.org/policy/guides/adopted/food.htm (accessed October 2012).

14. Shroff MR, Jones SJ, Frongillo EA et al. (2012) Policy instruments used by States seeking to improve school food environments. Am J Public Health 102, 222-229.

15. National Conference of State Legislatures (2011) Breastfeeding Laws. http://www.ncsl.org/issues-research/health/ breastfeeding-state-laws.aspx (accessed October 2012).

16. Jensen JD \& Smed S (2007) Cost-effective design of economic instruments in nutrition policy. Int J Behav Nutr Phys Act 4, 10.

17. Bastian A \& Coveney J (2012) Local evidenced-based policy options to improve food security in South Australia: the use of local knowledge in policy development. Public Health Nutr 15, 1497-1502.

18. Sabatier P (1988) An advocacy coalition framework of policy change and the role of policy-oriented learning therein. Policy Sci 2/213, 129-168.

19. Kingdon JW (2002) Agendas, Alternatives, and Public Policies. New York: Longman.

20. Leeman J, Sommers J, Vu M et al. (2012) An evaluation framework for obesity prevention policy interventions. Prev Chronic Dis 9, E120.

21. Lawrence M \& Swinburn B (2010) The role of policy in preventing childhood obesity. In Preventing Childhood Obesity: Evidence Policy and Practice, pp. 203-211 [E Waters, B Swinburn, J Seidell, et al. editors]. Chichester: Wiley-Blackwell.

22. Center for Public Health Nutrition (2010) Opportunities for Increasing Access to Healthy Foods in Washington: A Report for the Access to Healthy Foods Coalition. Seattle,
WA: University of Washington Center for Public Health Nutrition.

23. Kaphingst KM \& Story M (2009) Child care as an untapped setting for obesity prevention: state child care licensing regulations related to nutrition, physical activity, and media use for preschool-aged children in the United States. Prev Chronic Dis 6, A11.

24. Pomeranz JL \& Brownell KD (2011) Advancing public health obesity policy through state attorneys general. $\mathrm{AmJ}$ Public Health 101, 425-431.

25. Robert Wood Johnson Foundation (2010) Action Strategies Toolkit: A Guide for Local and State Leaders Working to Create Healthy Communities and Prevent Childhood Obesity. Princeton, NJ: Robert Wood Johnson Foundation.

26. Winterfeld A, Shinkle D \& Morandi L (2011) Reversing the Trend in Childhood Obesity: Policies to Promote Healthy Kids and Communities. Denver, CO: National Conference of State Legislatures.

27. Dillman DA (1999) Mail and Internet Surveys: The Tailored Design Method. Hoboken, NJ: John Wiley \& Sons, Inc.

28. Snowdon W, Potter JL, Swinburn B et al. (2010) Prioritizing policy interventions to improve diets? Will it work, can it happen, will it do harm? Health Promot Int 25, 123-133.

29. Snowdon W, Lawrence M, Schultz J et al. (2010) Evidenceinformed process to identify policies that will promote a healthy food environment in the Pacific Islands. Public Health Nutr 13, 886-892.

30. Gonzalez-Zapata L, Alvarez-Dardet C, Ortiz-Moncada R et al. (2009) Policy options for obesity in Europe: a comparison of public health specialists with other stakeholders. Public Health Nutr 12, 896-908.

31. Brescoll VL, Kersh R \& Bron KD (2008) Assessing the feasibility and impact of federal childhood obesity policies. Ann Am Acad Polit Sci 615, 178-194.

32. Shill J, Mavoa H, Allender S et al. (2012) Government regulation to promote healthy food environments - a view from inside state governments. Obes Rev 13, 162-173.

33. Jenkin G, Signal L \& Thomson G (2012) Nutrition policy in whose interests? A New Zealand case study. Public Health Nutr 15, 1483-1488.

34. Rivard C, Smith D, McCann SE et al. (2012) Taxing sugarsweetened beverages: a survey of knowledge, attitudes and behaviours. Public Health Nutr 15, 1355-1361.

35. Foltz JL, Harris DM \& Blanck HM (2012) Support among US adults for local and state policies to increase fruit and vegetable access. Am J Prev Med 43, 3 Suppl. 2, S102-S108.

36. Allender S, Gleeson E, Crammond B et al. (2012) Policy change to create supportive environments for physical activity and healthy eating: which options are the most realistic for local government? Health Promot Int 27, 261-274.

37. Gase LN, Kuo T, Dunet DO et al. (2011) Facilitators and barriers to implementing a local policy to reduce sodium consumption in the County of Los Angeles government, California, 2009. Prev Chronic Dis 8, A33.

38. Maestas C, Neeley GW \& Richardson LE Jr (2003) The state of surveying legislators: dilemmas and suggestions. State Politics Policy Q 3, 90-108. 